\title{
SOME ASPECTS OF TYPE II BURSTS
}

\author{
J. A. ROBERTS \\ Radiophysics Laboratory, Commonwealth Scientific and Industrial \\ Research Organization, Sydney, Australia
}

The outstanding bursts of radio emission from the sun, which are termed spectral type II or "slow-drift" bursts, are a rare phenomenon. Even at sunspot maximum the average rate of occurrence is only about one burst every 50 hours, so that a long series of observations is needed to define the characteristics of the bursts. The present study uses the records made over the past $5 \frac{1}{2}$ years with the 40 to $240 \mathrm{Mc} / \mathrm{s}$ Dapto radio spectrograph. During this period, 65 bursts of this type were recorded.

In bursts of spectral type II the frequency of maximum emission drifts slowly from high to low frequencies at a rate of about $1 \mathrm{Mc} / \mathrm{s}$ per second [1]. Often two drifting bands are present that are evidently the fundamental and second harmonic radiation of some nonlinear oscillation [2]. Furthermore, the individual harmonic bands are themselves sometimes split into two closely spaced ridges separated by about $10 \mathrm{Mc} / \mathrm{s}$. This effect, which was described earlier by Wild [3] and Wild, Murray, and Rowe [2], will be discussed in more detail below. It is also known that many type II bursts are preceded by a group of type III bursts occuring some minutes before the type II burst, but obviously related to it [4]. The more extensive observations reported here have confirmed this association, which occurs in approximately 60 per cent of cases.

\section{HERRING-BONE STRUCTURE IN TYPE II BURSTS}

In some cases the drifting bands of type II bursts are relatively smooth and continuous, but in other cases they contain considerable fine structure. This may take the form of narrow-band, short-duration bursts showing no frequency drift or drifting at a similar rate to the large scale features of the burst (Fig. 2(c)). Sometimes, however, the fine-structure elements are of broad bandwidth and short duration and drift at rates similar to type III bursts. Haddock [5] has already referred to this latter type of fine structure.

Fig. 1 contains examples of an interesting form of fine structure that appears on the record as a "herringbone" pattern. Here the slowly drifting band of the type II burst appears to be a source from which rapidly drifting elements extend toward both the high and the low frequencies. The features that drift toward the lower frequencies are very similar to type III bursts, although generally of rather shorter duration: those that drift toward the higher frequencies differ only in the sign of the rate of change of frequency. The 


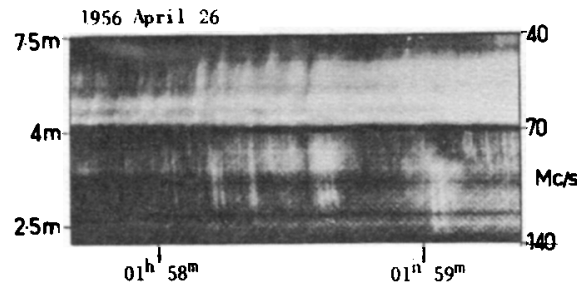

(a)

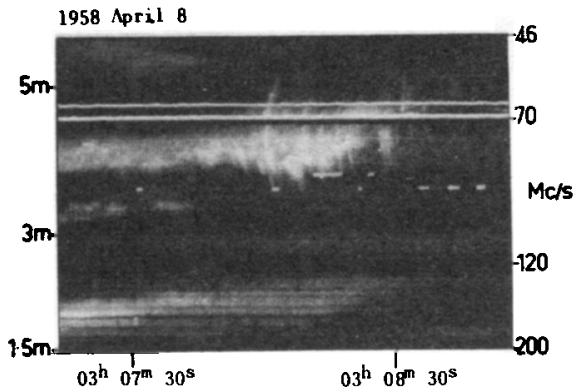

(c)

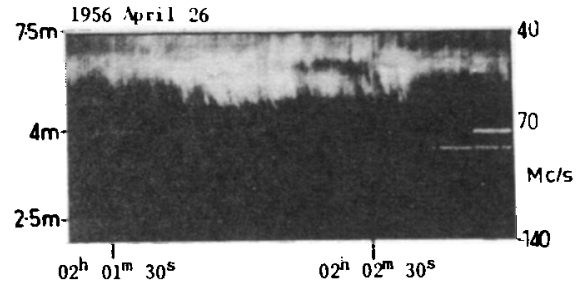

(b)

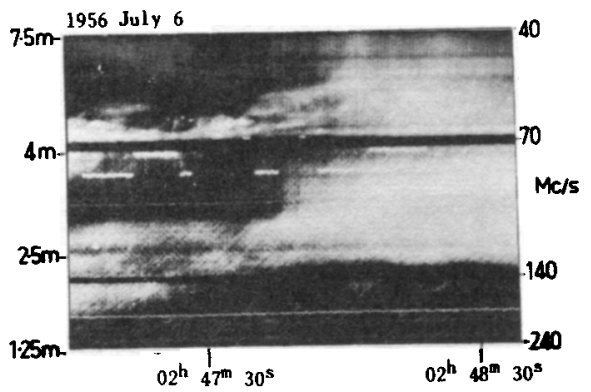

(d)

FIG. 1. Illustrating the herringbone structure in type II bursts. Only a small part of the burst is shown in each case : two sections of the burst on 1956 April 26 are included.

structure rarely forms such a dominant feature of the burst as in the example of Fig. 1(a) and $(b)$. Vestiges of the structure, however, in which the " fastdrift" elements extend over only a small range of frequencies, can be seen in perhaps 20 per cent of all type II bursts (Fig. 1(c)). Sometimes, as in the example in Fig. 1(d), the reverse-drift features are absent and the structure consists of a series of type III bursts starting from the type II band. This structure is very prominent in the example in Fig. 1(d); it can be seen in a less developed form in other examples.

The slowly drifting bands of the type II bursts are thought to be caused by disturbances traveling outward through the corona at speeds of about $500 \mathrm{~km} / \mathrm{second}$ and exciting plasma oscillations of successively lower frequencies as they pass into regions of lower electron density. Type III bursts are supposed to be produced in a similar manner but by disturbances moving with speeds of about $5 \times 10^{4} \mathrm{~km} / \mathrm{second}$. In terms of this theory the herringbone structure implies that the type II disturbances are sometimes the source of high speed (type III) ejections traveling both toward and away from the sun. Such secondary ejections are perhaps more easily understood if the type II disturbances are supersonic shock waves.

\section{HARMONIC STRUCTURE}

In 60 per cent of the type II bursts, a fundamental and a second harmonic band are clearly visible (Fig. 2). However, no example of a third harmonic is known, although in several cases one would have been detected had its 

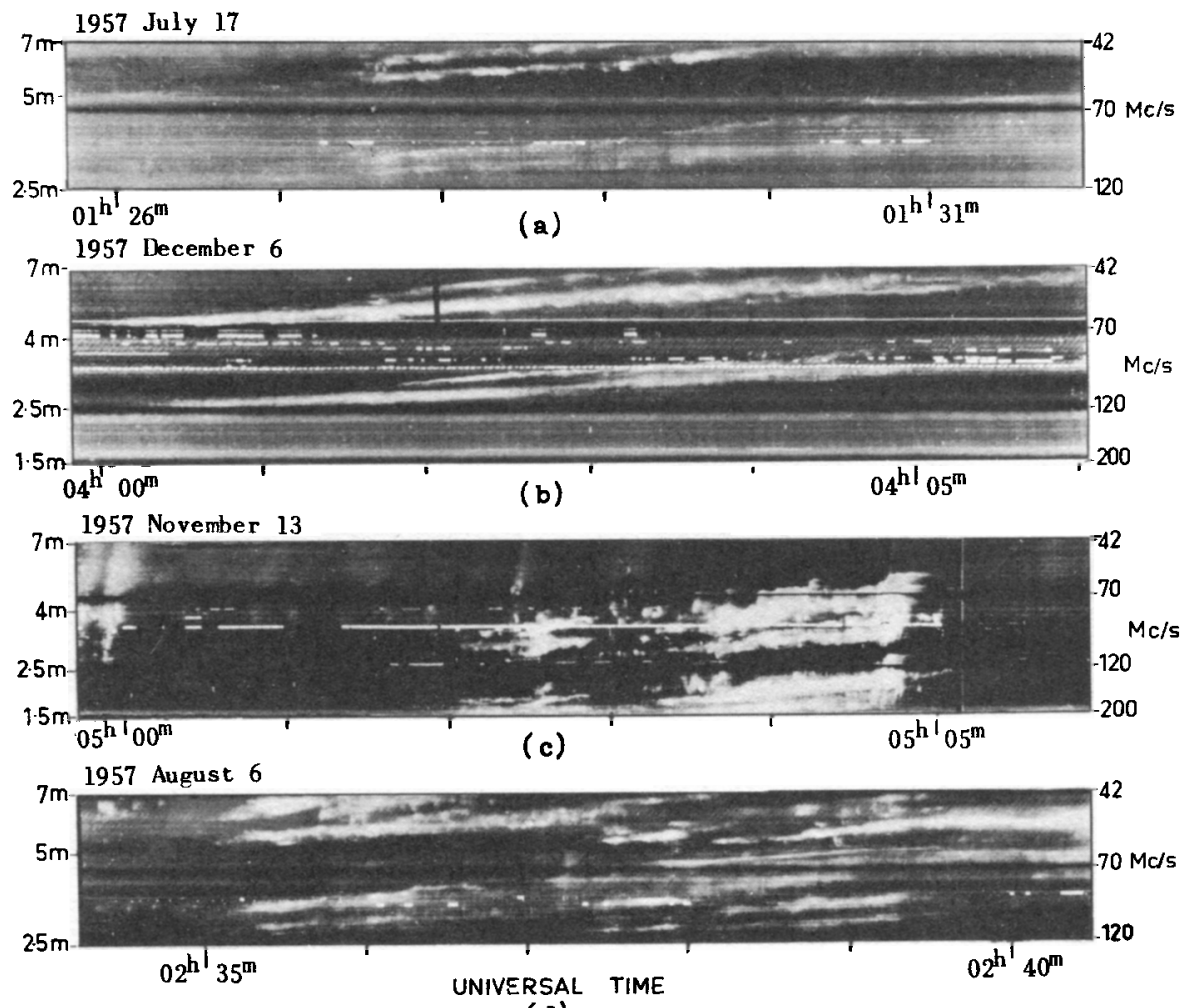

(d)

FIG. 2. Sample records of the dynamic spectra of type II bursts consisting of two harmonic bands, each of which is split into a pair of closely spaced ridges. In some cases not all of the burst is reproduced. Sharp features in these records, which lie along lines of constant frequency, are interfering signals.

intensity been 0.1 of that of the second harmonic. In one case (1956 April 25 ) it is possible that the second, third, and fourth harmonics are present, but this may be an example of a split band with a wide frequency separation.

The details in the fundamental and second harmonic bands usually correspond very closely, but there is never perfect agreement (Fig. 2). Wild, Murray, and Rowe [2] have already reported that the ratio of the peak frequencies is usually less than 2 : measurements of the frequency ratio of sharply defined features in 19 of the bursts in the present study yielded an approximately normal distribution with a median value of 1.95 and quartiles at 1.93 and 1.98. Wild et al. suggested that propagation conditions in the corona could cause this effect by preventing the low-frequency part of the fundamental band from reaching the earth. For a spherically symmetric corona the effect would be more pronounced for sources further away from the center of the disk: it was predicted that the frequency ratio would drop sharply with increasing radial distance and that the fundamental band would not be observed in bursts beyond $\sim 0.5 R_{\odot}$. 
The results shown in Fig. 3 do not bear out either of these predictions quantitatively. When the positions of the bursts are inferred from their associated optical events, it is found that both harmonics can be received from any part of the disk* (Fig. 3(a)). Furthermore, the frequency ratio shows no significant trend with radial distance and certainly does not decrease at the rate required by the theory (Fig. 3(b)). There is perhaps some indication in Fig. 3(a) that bursts with a single harmonic occur mainly near the limb. If this is taken to imply that the fundamental band is absent, then this result is in qualitative agreement with the predictions of the theory. Quantitative agreement may be possible if radiation in the fundamental band is strongly scattered by irregularities near the level of origin, so that it emerges in a wide cone and not in the very narrow beam predicted on the basis of a regular spherical corona. Since the refractive index for the fundamental band is close to zero at the level of origin, small variations in the electron density could produce considerable scattering.

(o)

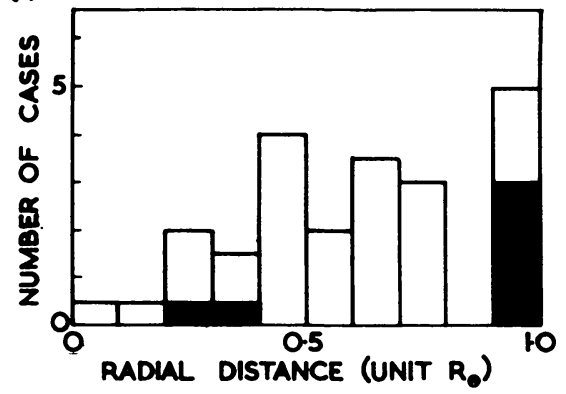

(b)

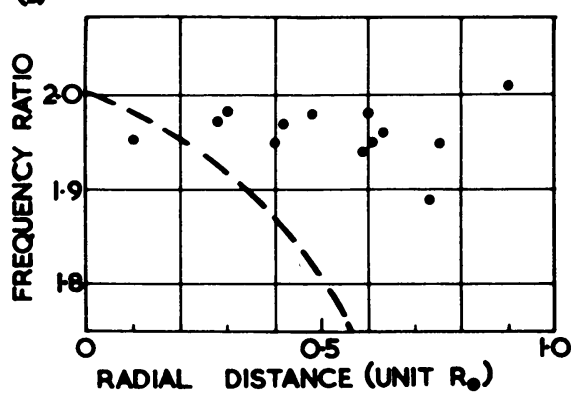

FIG. 3a. Distribution of type II bursts with radial distance. Unshaded regions refer to bursts with fundamental and second harmonic bands; shaded regions to bursts with a single band at such frequencies that harmonic structure could have been detected had it been present. The radial distances are those of the associated optical events, which in 19 cases were flares of class I or greater and in the remaining 3 cases were prominences ejected beyond the limb [6]. Fig. 3b. The mean ratio of the frequencies of corresponding features in the harmonic bursts. The curve shows the predicted ratio for a spherically symmetric corona [2].

\section{BAND SPLITTING}

The doubling of the fundamental and second harmonic bands referred to above is well illustrated by the examples in Fig. 2. This split-band structure is fairly common, occurring in about 20 per cent of cases. Often similar features appear in the two parts of the split band at close to the same time (Fig. 2). Because of this, and also in view of the narrow range of frequency separations observed at any one frequency (Fig. 4), it seems unlikely that the two parts are produced by two different disturbances. It seems more likely that they are produced by a single source radiating at two different frequencies.

* The trend seen in Fig. 3(a) for more bursts to occur at larger radial distances presumably arises from the increase with distance of the area of the (flareproducing) solar surface associated with a fixed interval of radial distance. 


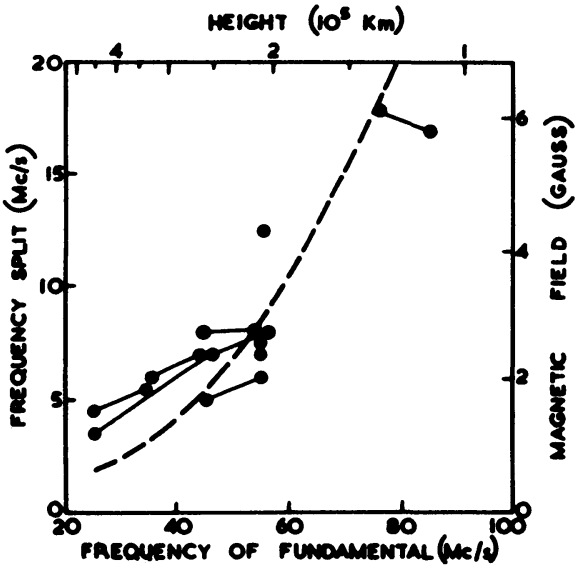

FIG. 4. The points show the frequency separation in split-bands, referred to the fundamental band. Points that are joined refer to the same burst. The dashed curve shows the variation of the gyro frequency (ordinate) with the coronal plasma frequency (abscissa) for a Chapman model sunspot of surface field 3000 gauss and with the Baumbach-Allen model of coronal electron densities. For this curve the axes may also be labeled as magnetic-field strength and height above the photosphere, as indicated on the diagram. Data from Smerd [7].
The magnitude of the frequency separation conforms with the splitting's being a Zeeman effect, as suggested by Wild [3]. This is illustrated by Fig. 4 which compares the measured frequency separations with the gyro frequency, $f_{H}$, above a large sunspot. If the identification as a Zeeman effect could be confirmed, this would provide a valuable method of measuring magnetic fields high in the corona in a region not accessible to optical observations.

Unfortunately the theory of the oscillation of a plasma in a magnetic field appears to be in a rather confused state: none of the suggested theories makes predictions completely in accord with the observations. According to some authors (e.g. Westfold [8]) such a plasma has three frequencies of oscillation corresponding to the zeros of the refractive index. These are the plasma frequency, $f_{0}$, and two frequencies which are approximately $f_{0} \pm \frac{1}{2} f_{H}$. In the fundamental band it is thought that the lowest of these could not

escape from the sun, so that a double band would be predicted in agreement with the observations. However, in the second harmonic all three frequencies should escape, which is contrary to the observations. Other authors (Gross [9], Sen [10]) have suggested that there are only two frequencies of oscillation, which are those for which the refractive index is very great. The frequencies predicted on this theory, however, are not close to one another and do not represent a splitting of the plasma level. It appears that a more complete theory is required to take account of the nature of the excitation: an extension of the work of Pines and Bohm [11] and Akhiezer and Sitenko [12] to include the case of a plasma in a magnetic field would seem desirable. Such an investigation should also examine the polarization of the emitted radiation, since the observed bands are substantially unpolarized.

\section{TYPE II BURSTS AND MAGNETIC STORMS}

A superposed epoch diagram of the magnetic variability on the earth following a type II burst on the sun shows the field as highly disturbed from 1 to 3 days after a burst (Fig. 5(a)). The probability that such a high value occurs by chance is about 1 in $10^{3}$. No such significant peak is found 
after flares of class 2 or 3 when these are not accompanied by type II bursts, nor after large type III events which are not associated with type II bursts. These results suggest that the occurrence of a type II burst is significant for the appearance of a magnetic storm on the earth. It should be emphasized, however that this is a statistical result and that in only a few cases is it possible with any certainty to associate a particular magnetic storm with a particular type II burst.

The curve given in Fig. 5(a) is very similar to that found by Dodson [13]
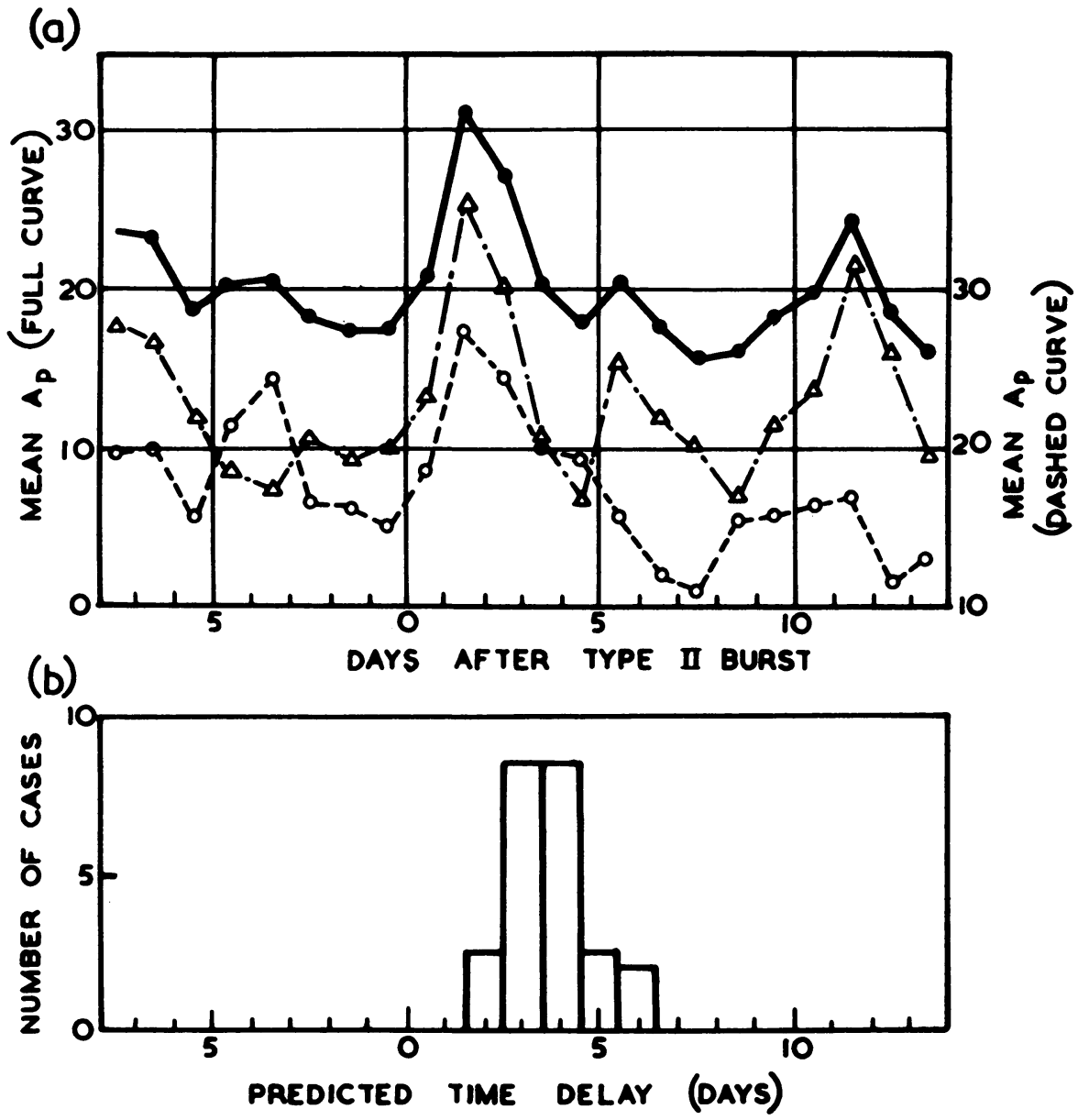

FIG. 5a. Superposed epoch diagram showing the mean value of the geomagnetic index $A_{p}$ on days before and after 60 type II bursts recorded in the period 1955 January to 1958 March. The dashed curves give the corresponding diagrams for the first 30 and the second 30 bursts separately. Note that these curves are plotted on a displaced scale of $A_{p}$. FIG. $5 b$. The predicted time delays inferred from the frequency drift rates in 24 of the type II bursts in which harmonic structure could be clearly recognized. It is assumed that the type II disturbances move radially outward through a Baumbach-Allen model corona and cause emission at the local coronal plasma frequency. 
for the magnetic variability after "major early bursts" at $200 \mathrm{Mc} / \mathrm{s}$. The present analysis, therefore, suggests that such events include a considerable proportion of bursts of spectral type II. Such a conclusion is not inconsistent with our knowledge of the time relationship of type II bursts and flares.

It is tempting to identify the disturbances producing type II bursts with the solar particles that are thought to produce the magnetic storms. The comparison given in Fig. 5 between the time delays of the magnetic disturbances and those inferred from the frequency drift rates of type II bursts suggests that either $(a)$ the disturbances producing type II bursts are mostly ejected at angles $>40^{\circ}$ to the radial direction, or $(b)$ the gradient of electron density in the corona is lower by a factor $\sim 2$ than that given by the BaumbachAllen model, or $(c)$ acceleration occurs subsequent to the emission of the type II radiation. It should be remembered, however, that the statistics on magnetic variability are small and that in the analysis referred to above Dodson found the maximum geomagnetic variability occurred 3 to 4 days after the radio burst and not 2 days as found here.

\section{ACKNOWLEDGMENTS}

The observations used in this study were made under the direction of $\mathrm{Mr}$. J. P. Wild as part of the Commonwealth Scientific and Industrial Research Organization's program for the study of the spectra of solar radio disturbances. Messrs. J. D. Murray, K. V. Sheridan, W. C. Rowe, M. Komesaroff, J. Joisce, and G. H. Trent assisted with various phases of the observational program. Thanks are due particularly 'to Mr. D. McClean, and also to Mrs. P. M. Hodgson and Misses P. Wilkins and J. A. Boorman for their assistance with the analysis of the results. I have to thank Dr. R. G. Giovanelli for data on flare observations at Sydney, and Messrs. J. P. Wild and S. F. Smerd and Dr. R. Q. Twiss for discussions on the interpretation of the observations.

\section{REFERENCES}

[1] Wild, J. P., and McCready, L. L. Aust. J. Sci. Res. A 3, 387, 1950.

[2] Wild, J. P., Murray, J. D., and Rowe, W. C. Aust. J. Phys. 7, 439, 1954.

[3] Wild, J. P., Aust. J. Sci. Res. A 3, 399, 1950.

[4] Wild, J. P., Roberts, J. A., and Murray, J. D. Nature, 173, 532, 1954.

[5] Haddock, F. T. Proc. I. R. E. 46, 3, 1958.

[6] Giovanelli, R. G., and Roberts, J. A. Aust. J. Phys. 11, 353, 1958.

[7] Smerd, S. F. Proc. I. E.E. 97 (III), 447, 1950.

[8] Westfold, K. C. Aust. J. Sci. Res. A 2, 169, 1949.

[9] Gross, E. P. Phys. Rev. 82, 232, 1951.

[10] Sen, H. K. Phys. Rev. 88, 816, 1952.

[11] Pines, D., and Bohm, D. Phys. Rev. 85, 338, 1952.

[12] Akhiezer, A. I., and Sitenko, A. G. Zh. Eksp. Teor. Fiz. 23, 161, 1952.

[13] Dodson, Helen W. Proc. I. R. E. 46, 149, 1958. 